\title{
Incremento de la seguridad de aprender mediante el aprendizaje de pares
}

\author{
asociados
}

\section{Enhancing the security of learning through the use of associated pairs}

\author{
Clara Nogueira-Prego, Alfredo Campos \\ Universidad de Santiago de Compostela
}

\begin{abstract}
Resumen
El objetivo principal de este estudio era examinar si la utilización de imágenes mentales y la capacidad de control de las mismas influyen en el recuerdo y en la seguridad en la respuesta $(\mathrm{CL}=$ Confidence level $)$. El grupo control memorizó la lista de pares de palabras mediante el método de repetición y el grupo experimental utilizó las imágenes mentales. El Gordon Test of Visual Imagery Control (GTVIC) midió la capacidad de control. Los resultados obtenidos confirman el efecto beneficioso de las imágenes mentales sobre el recuerdo y sobre la seguridad en la respuesta.
\end{abstract}

Palabras clave: aprendizaje mnemotécnico, imagen mental, memoria, metamemoria.

\section{Abstract}

The main goal of this study was to examine if the use of mental images and the ability to control them influence in the recall and confidence in response $(\mathrm{CL}=$ Confidence level). The control group memorized the word pairs list using the repetition method and the experimental group used the mental images. The Gordon Test of Visual Imagery Control (GTVIC) measured the control capability. The results confirm the beneficial effect of mental images on recall and on the sureness of response. Keywords: mnemonic learning, imagery, memory, metamemory.

El dicho de "una imagen vale más que mil palabras" hace referencia a la efectividad de una imagen en la transmisión de un mensaje que precisaría de muchas palabras para ser expresado. No obstante, este refrán también alude al hecho de que las imágenes resultan efectivas a la hora de recordar material verbal (Higbee, 1991). Las imágenes mentales actúan como mnemotecnias ya que ayudan a la memoria de manera inusual o artificial y promueven el aprendizaje del material y su posterior recuperación (Campos, 1998). La investigación acerca de la eficacia de las imágenes mentales en el aprendizaje de material verbal informa de un efecto positivo sobre el rendimiento. El recuerdo en serie, el recuerdo libre, el de pares de palabras, recuerdo con indicios o el reconocimiento se benefician del uso de las imágenes mentales cuando las palabras son altas en imaginabilidad (Richardson, 1980).

Una de las reglas mnemotécnicas más empleadas es la asociación de imágenes mentales, que consiste en la elaboración de una imagen mental en la que se incluyen cada uno de los elementos del material de estudio. Las investigaciones parecen concluir que, para garantizar la efectividad de dicha regla, es necesario que las imágenes sean: interactivas, extrañas y vivas (Higbee, 1991).

Un campo de estudio sobre el que ha girado buena parte de las investigaciones sobre imágenes mentales es el de las diferencias individuales en la capacidad de imagen, en concreto, en la capacidad para generar imágenes vivas (viveza de imagen) y para controlar su formación (control de imagen). En general, los autores informan de un efecto positivo de la capacidad de viveza (Camino, 2012, Campos \& Ameijide, 2011; Campos \& Fernández, 1995; Campos \& Pérez, 1997) y de control sobre el recuerdo (Campos, Rodríguez-Pinal \& Pérez-Fabello, 2013). En esta investigación se examinó la influencia de la mnemotecnia de imágenes mentales y la capacidad de control sobre el recuerdo.

Las imágenes mentales también afectan a la metamemoria, entendida ésta como el conocimiento y control que posee cada persona sobre el funcionamiento de su propia memoria, durante el registro, el almacenamiento y la recuperación de la información (González, 1997; Ruíz, 2008). El marco teórico más empleado en las investigaciones sobre metamemoria es el de Nelson y Narens (1990), quienes dentro de su modelo general de memoria, plantean que el estudio de la metamemoria se puede llevar a cabo a partir de los juicios que la persona realiza en 4 momentos del proceso de aprendizaje: a) antes del aprendizaje, la persona predice el grado de dificultad que le supondrá aprender los ítems presentados (Juicios de Facilidad de Aprender, $\mathrm{EOL}=$ Ease of Learning Judgements), b) durante la prueba o inmediatamente después de la adquisición pero antes de la prueba de recuperación, la persona evalúa el nivel de conocimiento que posee (Juicios de Aprendizaje, JOL= Judgements of Learning), c) durante la prueba de recuperación, la persona estima su rendimiento en una prueba de memoria posterior del mismo o distinto formato (Sensación de Saber o Impresión de Saber, FOK= Feeling of Knowing Judgements) y d) después de la recuperación del material de estudio, la persona emite juicios sobre el grado de seguridad que tiene de que sus respuestas sean correctas (Juicios de Confianza, CL= Confidence Level). 
Son escasos los estudios que examinan la relación entre la metamemoria y la mnemotecnia. Pérez-Fabello y Soto-Carballo (2015) encontraron correlaciones entre las puntuaciones en los test de imagen y los juicios metacognitivos de estudiantes de Bellas Artes y Campos, Pérez-Fabello y Gómez-Juncal (2007) encontraron que el control de imagen influía positivamente en el reconocimiento y en los juicios de confianza.

Una de las razones que explican el interés por el estudio de la metamemoria es su posible asociación funcional con el rendimiento memorístico (Díaz \& Rodrigo, 1988; Ruíz, 2008), que hace suponer que las variables que aumentan el rendimiento, como la mnemotecnia, también incrementan la estimación de los juicios (Campos \& Ameijide, 2011; Dunlosky \& Nelson, 1994). Esta premisa ha sido confirmada por las investigaciones que han informado de los beneficios que tiene sobre la metamemoria la utilización de la mnemotecnia de imágenes mentales. Ameijide y Campos (2015), Camino (2012), Campos y Ameijide (2014) y Shing, Werkle-Bergner, Li y Linderberger (2009) encontraron mayores niveles de seguridad en la respuesta cuando se empleaban las imágenes mentales como estrategia de estudio.

En este este estudio nos propusimos examinar el impacto de la mnemotecnia de imágenes mentales sobre el recuerdo y los juicios de confianza, y analizar los efectos del control de imagen sobre el recuerdo y los niveles de seguridad en la respuesta.

\section{Método}

\section{Participantes}

La investigación se realizó con 87 participantes (54 mujeres y 33 hombres), alumnos de $4^{\text {a }}$ de ESO y de $2^{\circ}$ de Bachillerato del colegio Las Esclavas del Sagrado Corazón de A Coruña, con una media de edad de 16.25 $(S D=1.13)$. Todos participaron de forma voluntaria, y se les garantizó la confidencialidad de sus datos, si bien, en ningún momento tuvieron que identificarse

\section{Instrumentos}

Se empleó una lista con 20 pares de palabras, seleccionadas al azar del libro de Valle (1998), con una puntuación alta en imaginabilidad (superior a 5.20). Estas mismas características también las poseían los 4 pares de palabras escogidos para el entrenamiento.

Para evaluar la capacidad de los participantes para controlar las imágenes mentales, se utilizó la versión española (Pérez- Fabello y Campos, 2004) del Gordon Test of Visual Imagery Control (GTVIC: Richardson, 1969). Esta prueba está conformada por 12 ítems en los que se les pide a los participantes que respondan a cada pregunta con un "sí", "no" o "no se". Posteriormente, a la hora de corregir los resultados del test, las respuestas se clasifican en una escala que va de 0 a 2 (donde 0 = "no", 1 = "inseguro" y 2 = "sí"). Las preguntas se refieren a si los participantes son capaces de imaginar un automóvil en varios colores, posiciones y estados de movimiento. Esta prueba fue validada con población española por Pérez-Fabello y Campos (2004), obteniendo un alfa de Cronbach de .69.

\section{Procedimiento}

Las palabras incluidas en la lista se seleccionaron de manera aleatoria de entre aquellas que presentaban puntuaciones altas en imaginabilidad de las publicadas por Valle (1998). Una palabra es considerada alta en viveza cuando tiene una puntuación superior a $5.20 \mathrm{o}$, lo que es lo mismo, 2/5 desviaciones típicas por encima de la media (Valle, 1998). De esta forma, se escogieron 48 palabras, 24 de las cuales se seleccionaron al azar como palabras estímulo y las otras 24 como palabras asociadas. A su vez, 4 de las palabras estímulo y 4 de las palabras asociadas fueron elegidas como palabras de entrenamiento.

La recogida de datos se llevó a cabo, de manera independiente con el alumnado de los dos cursos de $4^{\circ}$ de ESO y los dos cursos de $2^{\circ}$ de Bachillerato. Cada clase fue dividida en dos grupos. El grupo control formado por 41 participantes ( 17 hombres y 24 mujeres), tenía la tarea de aprender la lista de pares de palabras utilizando el método de repetición. Por su parte, el grupo experimental, formado por 46 participantes (16 hombres y 30 mujeres), debía memorizar los pares de palabras mediante la formación de imágenes mentales. De cara al análisis de los resultados, todos los participantes que emplearon el método de imágenes conformaron el grupo experimental y aquellos que utilizaron el de repetición, el grupo control.

Antes de la realización de las pruebas, se llevó a cabo una breve presentación de la tarea en la que se les pidió su colaboración voluntaria, recalcando el carácter anónimo de sus respuestas. Todos los estudiantes recibieron una carpeta con las instrucciones, la hoja de respuesta del entrenamiento, la hoja de respuesta de la prueba y el GTVIC. En las instrucciones, se les explicaba la tarea y se les solicitaba que indicaran su edad y su género. En las instrucciones para el grupo control se les pedía a los participantes que memorizaran una lista de palabras empleando el método de repetición, es decir, cada vez que apareciese un par de palabras debían repetirlas para memorizarlas. Se les informaba que, finalizada la proyección de las palabras, debían completar la hoja de respuestas añadiendo al lado de la primera palabra del par su palabra asociada. Una vez escrita la respuesta, se les solicitaba que puntuasen el grado de confianza que tenían de que su respuesta era correcta. Para ello, se les ofrecía una escala del 1 al 5, donde 1 indicaba mucha seguridad de que la respuesta era incorrecta, 5 mucha seguridad de que era correcta y 2 y 4 se referían a niveles medios de seguridad. Las instrucciones para el grupo experimental fueron iguales salvo en la explicación del método de aprendizaje, pues en este caso se les indicaba que debían formar una imagen en la que se incluyeran las dos palabras que conformaban el par en interacción. Este proceso debían de repetirlo con cada par de palabras, olvidándose de las anteriores hasta el final de la prueba. Las instrucciones relativas al nivel de confianza fueron idénticas para ambos grupos.

La lista de palabras fue proyectada por medio de un cañón asociado a un ordenador. Cada diapositiva contenía un par de palabras y fueron proyectadas a un 
ritmo de 1 cada 15 segundos. Primero se presentaron las palabras de entrenamiento $\mathrm{y}$, una vez proyectadas, todos los participantes escribieron en la hoja de respuestas de entrenamiento, al lado de la palabra estímulo, la palabra asociada y, en una escala del 1 al 5, el grado de confianza (CL) de que sus respuestas eran correctas. Las hojas de respuestas fueron iguales para el grupo control y el experimental, es decir, contenían las mismas palabras en el mismo orden.

Finalizado el entrenamiento, se procedió a la proyección de los 20 pares de palabras. Igual que en el entrenamiento, el ritmo de presentación fue de 15 segundos por diapositiva. Para la fase de recuperación, los participantes, en la hoja de respuestas, escribieron las palabras asociadas e indicaron su correspondiente nivel de confianza (CL) en una escala de 1 a 5 , donde 5 indicaba mucha seguridad de que la respuesta era correcta, 1 mucha seguridad de que la respuesta era incorrecta y 2 y 4 significaban niveles medios de confianza. Para realizar estas tareas contaron con un tiempo aproximado de dos minutos. Una vez terminada esta parte del estudio, procedieron a completar el Gordon Test of Visual Imagery Control.

De cara al análisis, los participantes fueron divididos en función del método de aprendizaje empleado (repetición y pares asociados) y de las puntuaciones en el GTVIC en relación a la media (altas y bajas puntuaciones en control de imagen).

\section{Resultados}

En primer lugar, se calculó la consistencia interna del Gordon Test of Visual Imagery Control, obteniéndose un alfa de Cronbach de .80, que coincide con el valor recomendado por Nunnally (1978) para la investigación básica. Las medias y desviaciones típicas de los individuos en el recuerdo se encuentran en la Tabla 1.

Tabla 1

Medias y Desviaciones Típicas de Recuerdo, en Función de la Estrategia de Aprendizaje y del Control de Imagen

Para comprobar si existían diferencias entre las medias de los grupos, se realizó un ANOVA de 2 (altos y bajos

\begin{tabular}{lcccccc}
\hline \multicolumn{7}{c}{ Estrategia de Aprendizaje } \\
\hline & \multicolumn{2}{c}{ Repetición } & \multicolumn{3}{c}{$\begin{array}{c}\text { Pares } \\
\text { Asociados }\end{array}$} \\
\cline { 2 - 8 } Gordon & $M$ & $S D$ & $M$ & $S D$ & $M$ & $S D$ \\
\hline $\begin{array}{l}\text { Altos en } \\
\begin{array}{l}\text { Control } \\
\text { Bajos en }\end{array}\end{array}$ & 11.17 & 6.45 & 14.45 & 4.60 & 12.89 & 5.76 \\
$\begin{array}{l}\text { Control } \\
\text { Total }\end{array}$ & 7.73 & 4.78 & 15.23 & 4.17 & 11.79 & 5.79 \\
& 10.24 & 6.19 & 14.67 & 4.45 & 12.59 & 5.75 \\
\hline
\end{tabular}

en GTVIC) x 2 (método de repetición y de pares asociados). Los resultados informan de que no hay diferencias significativas entre los individuos que puntuaron alto y bajo en control de imagen (altos y bajos en GTVIC), $F(1,83)=1.10, p=.30, \eta^{2} p=.01$, potencia $=$ .18. La estrategia de aprendizaje influyó de manera significativa en el recuerdo, $F(1,83)=17.99, p<.001, \eta_{p}^{2}$ $=.18$, potencia $=.99$. Los individuos que emplearon el método de pares asociados mediante imágenes mentales obtuvieron unas puntuaciones medias en recuerdo $(M=$ 14.67, $S D=4.45$ ) superiores a las de los individuos que utilizaron el método de repetición $(M=10.24, S D=$ 6.19). La interacción entre las dos variables: altas y bajas puntuaciones en GTVIC y método de aprendizaje (repetición e imágenes mentales), no resultaron significativas, $F(1,83)=2.75, p=.10, \eta^{2} p=.03$, potencia $=.37$.

Las medias y desviaciones típicas de las puntuaciones en los juicios de confianza aparecen recogidas en la Tabla 2. Para comparar la diferencia entre las medias de los individuos con puntuaciones altas y bajas en GTVIC, y entre los dos métodos de aprendizaje (repetición e imágenes) en el nivel de seguridad en la respuesta se efectuó un ANOVA de 2 (altos y bajos en GTVIC) x 2 (método de repetición y de imágenes). No se obtuvieron diferencias significativas entre los grupos que puntuaron alto y bajo en el GTVIC, $F(1,83)=1.39, p=.24, \eta_{p}^{2}=$ .02 , potencia $=.22$. Sin embargo, la diferencia de las puntuaciones entre método de repetición y de pares asociados mediante imágenes si resultó ser significativa, $F(1,83)=18.79, p<.001, \eta^{2}=.19$, potencia $=.99$. Los participantes que emplearon el método de imágenes mentales obtuvieron unas medias en los juicios de confianza $(M=75.78, S D=20.33)$ superiores a los que utilizaron el método de repetición $(M=53.10, S D=$ 29.86). La interacción entre los métodos de aprendizaje (repetición e imágenes mentales) y las puntuaciones en GTVIC (altas y bajas) no fue significativa, $F(1,83)=$ $1.60, p=.21, \eta^{2}=.02$, potencia $=.24$.

Tabla 2

Medias y Desviaciones Típicas de Juicios de Confianza, en Función de la Estrategia de Aprendizaje y del Control de Imagen

\section{Discusión}

En este estudio se ha examinado el impacto que tiene la

\begin{tabular}{|c|c|c|c|c|c|c|c|}
\hline \multirow{3}{*}{ Gordon } & & \multicolumn{4}{|c|}{ Estrategia de Aprendizaje } & \multirow{2}{*}{\multicolumn{2}{|c|}{ Total }} \\
\hline & & \multicolumn{2}{|c|}{ Repetición } & \multicolumn{2}{|c|}{$\begin{array}{c}\text { Pares } \\
\text { Asociados }\end{array}$} & & \\
\hline & & $M$ & $S D$ & $M$ & $S D$ & $M$ & $S D$ \\
\hline $\begin{array}{l}\text { Altos } \\
\text { Control }\end{array}$ & & 57.07 & 31.78 & 75.64 & 20.49 & 66.79 & 27.87 \\
\hline $\begin{array}{l}\text { Bajos } \\
\text { Control }\end{array}$ & en & 42.27 & 21.48 & 76.15 & 20.76 & 60.63 & 26.88 \\
\hline Total & & 53.10 & 29.86 & 75.78 & 20.33 & 65.09 & 27.58 \\
\hline
\end{tabular}

capacidad para controlar imágenes mentales y el método de aprendizaje de imágenes mentales interactivas en el aprendizaje de pares de palabras de alta imaginabilidad y en los juicios de confianza (CL).

Para evaluar la influencia del control de imagen, se utilizó el Gordon Test of Visual Imagery Control (GTVIC; Richardson, 1969) que demostró tener una adecuada consistencia interna según las recomendaciones realizadas por Nunnally (1978) para evaluar los coeficientes alfa de Cronbach. Estos resultados difieren de los de Pérez-Fabello y Campos (2004), quienes en la adaptación española del cuestionario informaron de una consistencia interna (.69) por debajo de los valores mínimos recomendados (.70). 
En esta investigación se observó que el control de imagen no era una variable influyente sobre el recuerdo. Estos resultados contradicen los encontrados por Campos, Amor y González (2002), Campos et, al. (1997, 2013), y Campos, González y Amor (2004). Campos, et al. (2014) informaron de los beneficios de la capacidad de control de imagen sobre el recuerdo productivo y reproductivo. La ausencia de correlaciones significativas entre las puntuaciones en el GTVIC y los juicios de confianza ya habían sido documentadas en el estudio de Pérez- Fabello y Soto-Carballo (2015), en el que sólo encontraron correlaciones significativas entre el GTVIC y los juicios de facilidad de aprendizaje y entre el GTVIC y los juicios de aprendizaje. En la investigación de Gómez- Juncal, Campos \& Pérez-Fabello (2003), tampoco se encontró que la capacidad para controlar imágenes mentales influyese en la seguridad de los juicios de reconocimiento.

La interacción entre las estrategias de estudio y el control de imagen tampoco demostró tener efectos significativos sobre el recuerdo, coincidiendo con los resultados de Campos, et al. (2013, 2014).

En nuestro estudio, los participantes que emplearon las imágenes mentales como estrategia de estudio recordaron más pares de palabras y estuvieron más seguros de sus respuestas que los que utilizaron el método de repetición. Este resultado está en la línea de estudios previos que demostraron los beneficios de la mnemotecnia visual frente al método de repetición (Campos \& Ameijide, 2011, 2014, 2015; Carney \& Levin, 2011; Kole \& Healy, 2013). La teoría de la Codificación Dual de Paivio (1979) permite explicar estos resultados ya que argumenta que lo que se estudia con imágenes mentales se procesa a nivel verbal y visual, reforzándose de este modo su aprendizaje frente al método de repetición en el que únicamente se procesa la información a nivel verbal.

Nuestros resultados respaldan la idea de que la mnemotecnia influye en el recuerdo y en la metamemoria (Campos \& Ameijide, 2014). Los participantes que utilizaron imágenes mentales durante su estudio se mostraron más seguros de sus respuestas que los que emplearon el método de repetición. Campos y Ameijide (2015) documentaron el efecto positivo de las imágenes mentales y de la palabra clave, en particular, en los juicios de confianza de personas mayores. Campos y Ameijide (2014) también informaron de mayores niveles de seguridad cuando los participantes empleaban el método de la palabra clave reforzada con dibujos. Con adolescentes, Ameijide y Campos (2015) encontraron que el método de la palabra clave reforzada con dibujos provocaba mayores niveles de confianza en las respuestas que el método de repetición.

Si bien los resultados obtenidos son significativos y coinciden con los de investigaciones previas, determinadas características de la metodología del estudio pueden limitar la generalización de las conclusiones. El estudio se llevó a cabo con un reducido número de participantes que compartían características socioculturales. Principalmente el hecho de que todos fueran estudiantes pudo afectar a las diferencias en el recuerdo entre ambas estrategias (imágenes mentales vs repetición), ya que los participantes de los dos grupos pueden tener un gran dominio sobre tareas de memorización.

La evaluación de la capacidad de imagen exclusivamente con un cuestionario que mide la capacidad de control, puede resultar insuficiente para conocer el papel que desempeña la capacidad de imagen en el rendimiento de tareas de recuerdo y en la seguridad de respuesta. La utilización de medidas de viveza y pruebas de rendimiento, unida a la evaluación de la capacidad de imagen, habría garantizado una mayor solidez en las conclusiones del estudio.

La selección de la seguridad en la respuesta como única variable metamnemónica impide la extrapolación de los hallazgos de este estudio al resto de juicios y, por tanto, ofrece una visión limitada del impacto de las imágenes mentales y la capacidad de control sobre la metamemoria.

Debido a que la línea que investiga la relación entre mnemotecnia y metamemoria es relativamente reciente sería conveniente realizar nuevos estudios que clarifiquen el tipo de relación que mantienen y las variables que inciden sobre ella. Estudiar a grupos poblacionales más amplios y heterogéneos puede contribuir a conocer el impacto de las diferencias individuales (género, edad, control de imagen, viveza...) en la eficacia de las imágenes mentales y en los juicios metamnemónicos. El hecho de que la utilización de imágenes mentales promueva la emisión de juicios de confianza positivos implica que los participantes son conscientes de los beneficios que sobre la memoria tiene la utilización de imágenes mentales y, de esta manera, los individuos pueden adoptar dicha estrategia como técnica habitual de aprendizaje y así lograr mejores resultados en pruebas de memorización. Tomados globalmente, nuestros resultados pueden ser una evidencia de la contribución de las creencias personales a la emisión de los juicios de confianza y pueden ser relevantes para entender cómo las personas juzgan su aprendizaje. Otros estudios ya han puesto de relieve la contribución de las creencias sobre los juicios metamnemónicos de facilidad para aprender (Mueller \& Dunlosky, 2017).

Por otra parte, puesto que nuestro estudio no respalda los resultados de las investigaciones precedentes en la eficacia del control de imagen en los juicios de confianza, se plantea la necesidad de profundizar en el impacto que ejerce la capacidad para formar imágenes mentales (viveza, control y las aptitudes visoespaciales) sobre la memoria y la metamemoria.

De cara a futuras investigaciones convendría explorar la influencia de las imágenes mentales en la exactitud entre los juicios de confianza y demás juicios metamnemónicos y el desempeño real. De encontrarse correlaciones, puesto que la utilización de imágenes es una habilidad susceptible de ser aprendida y practicada (Campos \& Pérez, 1996), se pondría de manifiesto la necesidad de promover la auto-reflexión y de introducir el entrenamiento en imágenes mentales en las escuelas para optimizar el proceso de enseñanza- aprendizaje. (Pérez- Fabello \& Soto- Carballo, 2015). 


\section{Referencias}

Ameijide, L. \& Campos, A. (2015). Confianza en la mnemotecnia de la palabra clave para aprender lenguas. Revista de Estudios e Investigación en Psicología y Educación, 1, 156-159. https://doi.org/10.17979/reipe.2015.0.01.915

Bower, G. H. (1970). Analysis of a mnemonic device. Modern psychology uncovers the powerful components of an ancient system for improving memory. American Scientist, 58, 496-510. http://www.jstor.org/stable/27829239

Camino, E. (2012). La mnemotecnia de la palabra clave favorece los juicios metamnemotécnicos positivos (Tesis doctoral). Universidad de Vigo, Ourense.

Campos, A. (Ed.) (1998). Imágenes mentales. Santiago de Compostela: Universidad de Santiago de Compostela.

Campos, A., \& Amejide, L. (2011). Exactitud de los Juicios de aprendizaje (JOLS) y mnemotecnia de la palabra clave en adultos y ancianos. International Journal of Psychological Research, 4, 64-71. http://dx.doi.org/10.21500/20112084.782

Campos, A., \& Ameijide, L. (2014). Mnemotecnia de la palabra clave con dibujos y juicios metamnemónicos de personas mayores. Revista Iberoamericana de Psicología y Salud, 5, 23-38.

Campos, A., \& Ameijide, L. (2015). Mnemotecnia y metamemoria en las personas mayores. Universitas Psychologica, 14, 57- 66.

http://dx.doi.org/10.11144/Javeriana.upsy14-1.mmpm

Campos, A., Amor, A. \& González, M. A. (2002). Presentation of keywords by means of interactive drawings. Spanish Journal of Psychology, 5, 102-109.

http://dx.doi.org/10.1017/S1138741600005874

Campos, A., \& Fernández, C. (1995). Imagen mnemónica y recuerdo de series de palabras. Adaxe, $11,27-33$.

Campos, A., González, M. A. \& Amor, A. (2004). Different strategies for keyword generation. Journal of Mental Imagery, 28 (3 y 4), 51-58.

Campos, A. \& Pérez, M. J. (1996). Viveza de imagen de pares asociados. Cognitiva, 2, 203-214.

Campos, A., \& Pérez, M. J. (1997). Mnemonic images and associated pair recall. Journal of Mental Imagery, 21, 73- 82.

Campos, A., Pérez-Fabello, M. J. \& Gómez-Juncal, R. (2007). Metamemory: effects of age and imaging control capacity. Imagination, Cognition and Personality, 26 , $179-190$. http://dx.doi.org/10.2190/J117-VK78-R211-8155

Campos, A., Rodríguez-Pinal, M. D., \& Pérez- Fabello, M. J. (2013). Aprendizaje del idioma gallego mediante la mnemotecnia de la palabra clave, en personas bilingües, altas y bajas en control de imagen. Revista de Investigación en Educación, 11, 50-59.

Campos, A., Rodríguez-Pinal, M. D., \& Pérez-Fabello, M. J. (2014). Receptive and productive recall with keywords mnemonics in bilingual students. Current Psychologhy, 33, 64-72. http://dx.doi.org/10.1007/s12144-013-9197-y
Carney, R. N. \& Levin, J. R. (2000). Fading mnemonic memories: Here's looking anew, again! Contemporary Educational Psychology, 25, 499-508. https://dx.doi.org/10.1006/ceps.1999.1035

Díaz, J. M. \& Rodrigo, M. J. (1989). Metamemoria y estrategias mnémicas en escolares. Infancia y Aprendizaje, 4 6,3-16. http://dx.doi.org/10.1080/02103702.1989.10822232

Dunlosky, J. \& Nelson, T. O. (1994). Does the sensitivity of judgements of learning (JOLs) to the effects of various study activities depend on when the JOLs occur? Journal of Memory and Language, 33, 545-565. https://dx.doi.org/10.1006/jmla.1994.1026

Gómez-Juncal, R., Campos, A., \& Pérez-Fabello, M. J. (2003). Imagen y edad en la seguridad de los juicios en la memoria de reconocimiento. Revista GalegoPortuguesa de Psicología e Educación, 10, 1007-1013.

González, A. (1997). Metamemoria y aprendizaje de textos. Estudios de Psicología, 58, 59-83. http://dx.doi.org/10.1174/021093997320954854

Higbee, K. L. (1998). Su memoria: Cómo funciona y cómo mejorarla. Barcelona: Ediciones Paidós.

Kole, J. A., \& Healy, A. F. (2013). Is retrieval mediated after repeated testing? Journal of Experimental Psychology: Learning, Memory, and Cognition, 39, 462-472. http://dx.doi.org/10.1037/a0028880

Mueller, M. L., \& Dunlosky, J. (2017). How beliefs can impact judgments of learning: Evaluating analytic processing theory with beliefs about fluency. Journal of Memory and Language, 93, 245- 258. http://dx.doi.org/10.1016/j.jml.2016.10.008

Nelson. T. O., \& Narens, L. (1990). Metamemory: A theoretical framework and some new findings. In G. H. Bower (Ed.), The psychology of learning and motivation (Vol. 26, pp. 125-173). New York: Academic Press.

Nunnally, J. C. (1978). Psychometric theory (2 $2^{\mathrm{a}}$ ed.). New York: McGraw-Hill.

Paivio, A. (1975). Imagery and long-term memory. En A. Kennedy y A. Wilkes (Eds.), Studies in long term memory (pp. 57-88). London: Wiley.

Pérez-Fabello, M. J. \& Campos, A. (2004). Factor structure and internal consistency of the Spanish version of the Gordon Test of Visual Imagery Control. Psychological Reports, 94, 761-766. http://dx.doi.org/10.2466/pr0.94.3.761-766

Pérez-Fabello, M. J. \& Soto-Carballo, J. (2015). En entrenamiento en juicios metamnemónicos y su implicación en el rendimiento académico. Revista de Estudios e Investigación en Psicología y Educación, 1, 138141. http://dx.doi.org/10.17979/reipe.2015.0.01.671

Richardson, A. (1969). Mental imagery. London: Routledge \& Kagan Paul.

Richardson, A. (1980). Mental imagery and human memory. London: MacMillan.

Ruíz, M. (2004). Las dos caras de la memoria. Madrid: Pearson.

Shing, Y. L., Werkle-Bergner, M., Li, S. \& Lindenberger, U. (2009). Commiting memory errors with high confidence: Older adults do but children 
don`t. Memory, 17, (2), 169-179. http://dx.doi.org/10.1080/09658210802190596

Valle Arrollo, F. (1998). Normas de imaginabilidad. Oviedo: Servicio de Publicaciones de la Universidad de Oviedo. 